\title{
Analysis of the Performance and Sensitivity of an Eigenspace-Based Interference Canceler
}

\author{
Ju-Hong Lee and Cheng-Chou Lee
}

\begin{abstract}
Eigenspace-based interference cancelers (EIC's) possess the advantages of providing maximal suppression of interference with fast convergence over conventional adaptive beamformers. However, the performance and sensitivity to steering angle error of EIC's have not been analyzed due to the use of a signal blocking matrix. We first present a technique to construct a positive definite matrix based on the signal blocking matrix and then use this matrix to compensate the effect of the signal blocking matrix on the sensor noise received by an EIC. Therefore, the interference subspace required for finding the optimal weight vector can be obtained using conventional eigenvalue decomposition (EVD). Moreover, the performance and sensitivity to the steering angle error of the EIC can be analyzed. Simulation examples are provided for confirming the theoretical results.
\end{abstract}

Index Terms-Adaptive antennas, electromagnetic interference.

\section{INTRODUCTION}

$\mathbf{T}$ ECHNIQUES for achieving the purpose of maximizing the rejection of interference regardless of the interference-to-noise ratio (INR) when processing array data by using adaptive interference cancelers have been reported in [1]-[6]. Notable among them is the one of [4] where an eigenanalysis interference canceler (EIC) with fast convergence speed using a uniform linear array (ULA) was presented. The optimal weight vector is computed by maximizing the output signal-to-background noise ratio subject to a constraint of orthogonality to the interference subspace (IS). The IS is obtained through the generalized eigenvalue decomposition (GEVD) of the correlation matrix of the data vector at the output of an appropriately designed blocking processor which blocks the desired signal from the received data vector.

Due to the use of a signal blocking matrix, the noise component contained in the blocked data vector is no longer spatially white. Therefore, finding the IS for computing the optimal weight vector generally requires a complicated GEVD. Moreover, it is not an easy task to analyze the performance and sensitivity to steering angle error of an EIC. Thus, in the literature, there are practically no papers considering the analysis of the performance and sensitivity of an EIC. In this paper, we present a technique to construct a positive definite matrix from the signal blocking matrix of an EIC. The effect of the signal blocking matrix on the spatially white noise component received

Manuscript received February 7, 1996; revised April 15, 1997. This work was supported by the National Science Council under Grant NSC85-2213-E002-008 and Grant NSC89-2213-E002-084.

The authors are with Department of Electrical Engineering, National Taiwan University, Taipei 106, Taiwan.

Publisher Item Identifier S 0018-926X(00)04372-6. by the EIC is then eliminated by adding the matrix to the correlation matrix of the blocked data vector. This results in that the IS required for computing the optimal weight vector of an EIC can be obtained by performing conventional EVD instead of any complicated GEVD. Moreover, it is shown that the EIC's performance and sensitivity to steering angle error can be analyzed based on the proposed technique. We present the analysis of the performance of the EIC in terms of the expectation of the output signal-to-interference plus noise ratio (SINR). As to the analysis of sensitivity to steering angle error, the theoretical results show that the EIC's performance is considerably deteriorated even if there is a small steering angle error. Increasing the order of the signal blocking matrix can alleviate this performance degradation. Moreover, the breakdown thresholds for the EIC's performance in the presence of two interferers with two extreme correlation cases are derived, respectively.

This paper is organized as follows. Section II briefly describes the principle of a conventional EIC. In Section III, we present the technique for constructing a positive definite matrix to eliminate the effect of the signal blocking matrix on the received data vector. Based on the proposed technique, the analysis of the EIC's statistical performance is presented in Section IV. We evaluate the EIC's sensitivity to steering angle error in Section $\mathrm{V}$. The performance breakdown thresholds are also derived for the cases of two interferers with two extreme correlation situations. Simulation examples for illustration and confirmation are included in Section VI. Finally, Section VII concludes the paper.

\section{Principle of a Conventional EIC}

Consider an $M$-sensor linear array with interelement spacing equal to $d$ illuminated by $P$ narrow-band signal sources from the distinct direction angles $\theta_{i}, i=1,2, \cdots, P$. Let the response of the $m$ th sensor to a signal with unit amplitude and a direction angle $\theta_{i}$ be given by $\exp \left(j(m-1) u_{i}\right)$, where $j=\sqrt{-1}, u_{i}=$ $2 \pi d \sin \left(\theta_{i}\right) \lambda$, and $\lambda$ is the wavelength of the signal sources. The received signal at the $m$ th sensor can be expressed as

$$
x_{m}(t)=\sum_{i=1}^{P} s_{i}(t) e^{j(m-1) u_{i}}+n_{m}(t)
$$

where $s_{i}(t)$ denotes the complex amplitude of the $i$ th signal impinging on the array with direction angle $\theta_{i}, n_{m}(t)$ the spatially white sensor noise with power $\pi_{n}$ received by the $m$ th sensor. Both the signal and sensor noise are assumed to be independent 
and zero-mean stationary Gaussian random processes. In vector form, the received data vector is given by

$$
\boldsymbol{x}(t)=\sum_{i=1}^{P} \boldsymbol{a}\left(u_{i}\right) s_{i}(t)+\boldsymbol{n}(t)=A \boldsymbol{s}(t)+\boldsymbol{n}(t)
$$

where the response vector of the $i$ th signal $\boldsymbol{a}\left(u_{i}\right)=$ $\left[1 \exp \left(j u_{i}\right) \cdots \exp \left(j(M-1) u_{i}\right)\right]^{T}$, the noise vector $\boldsymbol{n}(t)=$ $\left[\begin{array}{llll}n_{1}(t) & n_{2}(t) & \cdots & n_{M}(t)\end{array}\right]^{T}$, the signal source vector $s(t)=\left[\begin{array}{llll}s_{1}(t) & s_{2}(t) & \cdots & s_{P}(t)\end{array}\right]^{T}$, and the response matrix of the signal sources $A=\left[\begin{array}{llll}\boldsymbol{a}\left(u_{1}\right) & \boldsymbol{a}\left(u_{2}\right) & \cdots & \boldsymbol{a}\left(u_{P}\right)\end{array}\right]$. The superscript $T$ denotes the transpose operation.

Assume the direction angle of the desired signal is $\theta_{1}$. Based on the principle of the EIC presented in [4], a blocking matrix $\boldsymbol{B}$ is appropriately designed and used as the block processor in order to block the desired signal from the received data vector. Let the order of $\boldsymbol{B}$ be $q$ and a $(q+1) \times 1$ vector $\boldsymbol{b}=\left[\begin{array}{llll}b_{0} & b_{1} & \cdots & b_{q}\end{array}\right]^{T}$ be defined according to the following manner:

$$
\left(z-e^{j u_{1}}\right)^{q}=\sum_{r=0}^{q}(-1)^{q-r}\left(\begin{array}{l}
q \\
r
\end{array}\right) e^{j(q-r) u_{1}} z^{r}=\sum_{r=0}^{q} b_{r}^{*} z^{r}
$$

where the superscript $*$ denotes the complex conjugate. Construct an $M \times 1$ vector $\tilde{\boldsymbol{b}}=\left[\begin{array}{ll}\boldsymbol{b}^{T} & \boldsymbol{o}_{M-q-1}\end{array}\right]^{T}$, where $\boldsymbol{o}_{M-q-1}$ is a $1 \times(M-q-1)$ row vector with zero elements. Thus the signal blocking matrix with order $q$ can be expressed as

$$
\boldsymbol{B}=\left[\begin{array}{llllll}
\tilde{\boldsymbol{b}} & \tilde{\boldsymbol{I}}_{M} \tilde{\boldsymbol{b}} & \tilde{\boldsymbol{I}}_{M}^{2} \tilde{\boldsymbol{b}} & \cdots & \tilde{\boldsymbol{I}}_{M}^{M-q-1} \tilde{\boldsymbol{b}}
\end{array}\right]
$$

where $\tilde{\boldsymbol{I}}_{M}=\left[\begin{array}{lllll}\boldsymbol{u}_{2} & \boldsymbol{u}_{3} & \cdots & \boldsymbol{u}_{M} & \boldsymbol{u}_{1}\end{array}\right]$ represents a cyclic-shifting matrix with element $\boldsymbol{u}_{i}$ given by the $i$ th column vector of an $M \times M$ identity matrix. Based on $\boldsymbol{a}\left(u_{i}\right)=\left[\begin{array}{llll}1 & \exp \left(j u_{i}\right) & \cdots & \exp \left(j(M-1) u_{i}\right)\end{array}\right]^{T}$ and (3), we have

$$
B^{H} \boldsymbol{a}\left(u_{i}\right)=\nu_{i} \boldsymbol{a}_{q}\left(u_{i}\right)
$$

where $\boldsymbol{a}_{q}\left(u_{i}\right)=\left[1 \exp \left(j u_{i}\right) \cdots \exp \left(j(M-q-1) u_{i}\right)\right]^{T}$ and the blocking factor $\nu_{i}$ associated with the $i$ th signal source is given by

$$
\nu_{i}=\sum_{r=0}^{q} b_{r}^{*} e^{j r u_{i}}=\left(e^{j u_{i}}-e^{j u_{1}}\right)^{q} .
$$

The data vector at the output of the signal blocking matrix $B$ is then given by

$$
\boldsymbol{x}_{b}(t)=\boldsymbol{B}^{H} \boldsymbol{x}(\boldsymbol{t})=\boldsymbol{A}_{b} \boldsymbol{D}_{b} \boldsymbol{s}(t)+\boldsymbol{B}^{H} \boldsymbol{n}(t)=A_{b} \boldsymbol{s}_{b}(t)+\boldsymbol{n}_{b}(t)
$$

where $A_{b} \quad=\left[\begin{array}{llll}\boldsymbol{a}_{q}\left(u_{1}\right) & \boldsymbol{a}_{q}\left(u_{2}\right) & \cdots & \boldsymbol{a}_{q}\left(u_{P}\right)\end{array}\right]$ is the corresponding response matrix and $D_{b}=\operatorname{diag}\left\{\nu_{1}, \quad \nu_{2}, \cdots, \quad \nu_{P}\right\}$. Since $\nu_{1}=0$, (7) can be rewritten as

$$
\boldsymbol{x}_{b}(t)=\boldsymbol{A}_{I} \boldsymbol{D}_{I} \boldsymbol{s}_{I}(t)+\boldsymbol{n}_{b}(t)
$$

where $A_{I}=\left[\begin{array}{llll}\boldsymbol{a}_{q}\left(u_{2}\right) & \boldsymbol{a}_{q}\left(u_{3}\right) & \cdots & \boldsymbol{a}_{q}\left(u_{P}\right)\end{array}\right], \boldsymbol{D}_{I}=$ $\operatorname{diag}\left\{\nu_{2}, \nu_{3}, \cdots, \nu_{P}\right\}, \quad$ and $\quad \boldsymbol{s}_{I}(t)=$ $\left[\begin{array}{llll}s_{2}(t) & s_{3}(t) & \cdots & s_{\Gamma}(t)\end{array}\right]^{T}$. It follows from that the ensemble correlation matrix of $\boldsymbol{x}_{b}(t)$ is given by

$$
\boldsymbol{R}=E\left\{\boldsymbol{x}_{b}(t) \boldsymbol{x}_{b}(t)^{H}\right\}=\boldsymbol{A}_{I} \boldsymbol{\Psi}_{I} A_{I}^{H}+\pi_{n} \boldsymbol{B}^{H} \boldsymbol{B}
$$

where $\boldsymbol{\Psi}_{I}=E\left\{\boldsymbol{D}_{I} \boldsymbol{s}_{I}(t) \boldsymbol{s}_{I}(t)^{H} \boldsymbol{D}_{I}^{H}\right\}=\boldsymbol{D}_{I} \boldsymbol{\Phi}_{I} D_{I}^{H}$ with $\boldsymbol{\Phi}_{I}=$ $E\left\{\boldsymbol{s}_{I}(t) \boldsymbol{s}_{I}(t)^{H}\right\}$. Equation (9) reveals that to find the required IS from $\boldsymbol{R}$, one must perform the GEVD of $\boldsymbol{R}$ given that the noise component $\boldsymbol{n}_{b}(t)$ of $\boldsymbol{x}_{b}(t)$ is no longer spatially white and has correlation matrix $\pi_{n} \boldsymbol{B}^{H} \boldsymbol{B}$. After performing the GEVD of (9), we have the following relationship for the resulting generalized eigenvectors ( $g$-vectors) $\boldsymbol{g}_{i}$ and generalized eigenvalues ( $g$-values) $\lambda_{i}$

$$
\boldsymbol{R g}_{i}=\lambda_{i} B^{H} \boldsymbol{B} \boldsymbol{g}_{i}
$$

where $\lambda_{1} \geq \lambda_{2} \geq \cdots \geq \lambda_{P-1}>\lambda_{P}=\cdots=\lambda_{M-q}=\pi_{n}$. Using the $g$-vectors $\boldsymbol{g}_{i}$, we construct two matrices as follows: $\boldsymbol{G}_{I}=\left[\boldsymbol{g}_{1}, \boldsymbol{g}_{2}, \cdots, \boldsymbol{g}_{P-1}\right]$ and $\boldsymbol{G}_{R}=\left[\boldsymbol{g}_{P}, \boldsymbol{g}_{P+1}, \cdots, \boldsymbol{g}_{M-q}\right]$. Then, it is easy to show that the matrix $\boldsymbol{A}_{I}$ and $\boldsymbol{B}^{H} \boldsymbol{B} \boldsymbol{G}_{I}$ span the same subspace which is orthogonal to the subspace spanned by $\boldsymbol{G}_{R}$, i.e., range $A\left\{\boldsymbol{A}_{I}\right\}=\operatorname{range}\left\{\boldsymbol{B}^{H} \boldsymbol{B} \boldsymbol{G}_{I}\right\} \perp \operatorname{range}\left\{\boldsymbol{G}_{R}\right\}$.

As presented in [4], the criterion of an optimal EIC with reduced aperture size $M-q$ is to maximize the output signal-tobackground noise ratio subject to a constraint of orthogonality to the IS. If $A_{I}$ is known, the criterion is given by

$$
\text { Maximize } \frac{\left|\boldsymbol{w}^{H} \boldsymbol{a}_{q}\left(u_{1}\right)\right|^{2}}{\boldsymbol{w}^{H} \boldsymbol{w}} \quad \text { subject to } \boldsymbol{w}^{H} \boldsymbol{A}_{I}=0
$$

and the optimal weight vector is given by

$$
\boldsymbol{w}_{o}=\left(\boldsymbol{I}-\boldsymbol{A}_{I}\left(\boldsymbol{A}_{I}^{H} A_{I}\right)^{-1} \boldsymbol{A}_{I}^{H}\right) \boldsymbol{a}_{q}\left(u_{1}\right)
$$

where $I$ denotes the $(M-q) \times(M-q)$ identity matrix. In practice, $A_{I}$ is unknown and $\boldsymbol{w}^{H} \boldsymbol{A}_{I}=0$ can be replaced by $\boldsymbol{w}^{H} \boldsymbol{B}^{H} \boldsymbol{B} \boldsymbol{G}_{I}=\mathbf{0}$ since range $\left\{\boldsymbol{A}_{I}\right\}=\operatorname{range}\left\{\boldsymbol{B}^{H} \boldsymbol{B} \boldsymbol{G}_{I}\right\}$. Accordingly, the optimal weight vector $\boldsymbol{w}_{O}$ becomes

$$
\begin{aligned}
\boldsymbol{w}_{o}= & \left(\boldsymbol{I}-\left(\boldsymbol{B}^{H} \boldsymbol{B} \boldsymbol{G}_{I}\right)\left[\left(\boldsymbol{B}^{H} \boldsymbol{B} \boldsymbol{G}_{I}\right)^{H}\left(\boldsymbol{B}^{H} \boldsymbol{B} \boldsymbol{G}_{I}\right)\right]^{-1}\right. \\
& \left.\cdot\left(\boldsymbol{B}^{H} \boldsymbol{B} \boldsymbol{G}_{I}\right)^{H}\right) \boldsymbol{a}_{q}\left(u_{1}\right) .
\end{aligned}
$$

From (9), (10), and (13), we note that to evaluate the performance and sensitivity to steering angle of the EIC is very difficult and, hence, there are practically no papers considering this problem.

\section{EIC Using THE PRoposed TeChNiQUe}

In this section, we present an EIC based on a proposed technique to alleviate the difficulty described above. From (9), it is obvious that the difficulty is induced due to the effect of the signal blocking matrix $\boldsymbol{B}$ on the received sensor noise. To eliminate this effect, a technique is developed as follows. For the sake of simplicity, the notation $H T\left\{c_{1}, c_{2}, \cdots, c_{m}\right\}$ is used to denote an $m \times m$ Hermitian and Toeplitz matrix with its first row given by the row vector $\left[\begin{array}{llll}c_{1} & c_{2} & \cdots & c_{m}\end{array}\right]$. Using (3) and (4), it can be shown that $\boldsymbol{B}^{H} \boldsymbol{B}$ is given by

$$
\boldsymbol{B}^{H} \boldsymbol{B}=H T\left\{\epsilon_{0}, \epsilon_{1}, \cdots, \epsilon_{M-q-1}\right\}, \quad \text { with } \epsilon_{i}=\sum_{r=0}^{q-i} b_{r+i}^{*} b_{r} .
$$

Next, we construct an $(M-q+i) \times 1$ vector as follows:

$$
\boldsymbol{f}(d, i)=\left[\begin{array}{llll}
1 & \boldsymbol{o}_{i-1} & j^{d} & \boldsymbol{o}_{M-q-1}
\end{array}\right]^{T}
$$


where $i=1,2, \cdots, M-q-1$ and $d$ is an integer. From (15), an $(M-q+i) \times(M-q)$ matrix is constructed as follows:

$$
\begin{aligned}
\boldsymbol{F}(d, i)= & {\left[\begin{array}{lll}
f(d, i) & \tilde{\boldsymbol{I}}_{M-q+i} \boldsymbol{f}(d, i) & \tilde{\boldsymbol{I}}_{M-q+i}^{2} f(d, i) \\
& \tilde{\boldsymbol{I}}_{M-q+i}^{M-q-1} \boldsymbol{f}(d, i)
\end{array}\right] }
\end{aligned}
$$

where $\tilde{\boldsymbol{I}}_{M-q+i}$ is the $(M-q+i) \times(M-q+i)$ cyclic-shifting matrix. Using (15) and (16), we have

$$
\begin{aligned}
\boldsymbol{\Gamma}(d, i) & =\boldsymbol{F}(d, i)^{H} \boldsymbol{F}(d, i) \\
& =H T\left\{2, \boldsymbol{o}_{i-1},(-j)^{d}, \boldsymbol{o}_{M-q-1-i}\right\} .
\end{aligned}
$$

From (17), it is obvious that $\boldsymbol{\Gamma}(d, i)$ is positive definite, Hermitian, and Toeplitz. Moreover, it is easy to show that

$$
\begin{aligned}
\left|\operatorname{Re}\left\{\epsilon_{i}\right\}\right| \boldsymbol{\Gamma}\left(2 \operatorname{sgn}\left(\operatorname{Re}\left\{\epsilon_{i}\right\}\right), i\right)+\left|\operatorname{Im}\left\{\epsilon_{i}\right\}\right| \boldsymbol{\Gamma} \\
\quad \cdot\left(2 \operatorname{sgn}\left(\operatorname{Im}\left\{\epsilon_{i}\right\}\right)-1, i\right) \\
=H T\left\{2\left(\left|\operatorname{Re}\left\{\epsilon_{i}\right\}\right|+\left|\operatorname{Im}\left\{\epsilon_{i}\right\}\right|\right), \boldsymbol{o}_{i-1},-\epsilon_{i}, \boldsymbol{o}_{M-q-1-i}\right\}
\end{aligned}
$$

for $i=1,2, \cdots, M-q-1$, where $\operatorname{Re}\{x\}$ and $\operatorname{Im}\{x\}$ denote the real and imaginary parts of $x$, respectively. $\operatorname{sgn}(x)=1$ if $x>0$, and $=0$, otherwise. Finally, we construct a positive definite matrix as follows:

$$
\begin{gathered}
\boldsymbol{\Omega}=\sum_{i=1}^{M-q-1}\left(\left|\operatorname{Re}\left\{\epsilon_{i}\right\}\right| \boldsymbol{\Gamma}\left(2 \operatorname{sgn}\left(\operatorname{Re}\left\{\epsilon_{i}\right\}\right), i\right)+\left|\operatorname{Im}\left\{\epsilon_{i}\right\}\right| \boldsymbol{\Gamma}\right. \\
\left.\cdot\left(2 \operatorname{sgn}\left(\operatorname{Im}\left\{\epsilon_{i}\right\}\right)-1, i\right)\right) .
\end{gathered}
$$

Summing (14) and (19) thus yields a diagonal matrix as follows:

$$
\begin{aligned}
\boldsymbol{B}^{H} \boldsymbol{B}+\boldsymbol{\Omega} & =\left(\epsilon_{0}+2 \sum_{i=1}^{M-q-1}\left(\left|\operatorname{Re}\left\{\epsilon_{i}\right\}\right|+\left|\operatorname{Im}\left\{\epsilon_{i}\right\}\right|\right)\right) \boldsymbol{I} \\
& =\kappa \boldsymbol{I}
\end{aligned}
$$

where $\kappa$ denotes the proportional constant.

Based on (20), the effect of the signal blocking matrix on the received sensor noise can be eliminated by taking the following matrix:

$$
\boldsymbol{R}_{w}=\boldsymbol{R}+\pi_{n} \boldsymbol{\Omega}=\boldsymbol{A}_{I} \boldsymbol{\Psi}_{I} \boldsymbol{A}_{I}^{H}+\kappa \pi_{n} \boldsymbol{I}
$$

as a correlation matrix to replace the original correlation matrix $\boldsymbol{R}$. Accordingly, performing the EVD on $\boldsymbol{R}_{w}$ yields

$$
\boldsymbol{R}_{w} \boldsymbol{e}_{i}=\gamma_{i} \boldsymbol{e}_{i}
$$

where $\gamma_{1} \geq \gamma_{2} \geq \cdots \geq \gamma_{P-1}>\gamma_{P}=\cdots=\gamma_{M-q}=\kappa \pi_{n}$. Let the matrices $\boldsymbol{E}_{I}=\left[\begin{array}{llll}\boldsymbol{e}_{1} & \boldsymbol{e}_{2} & \cdots & \boldsymbol{e}_{P-1}\end{array}\right]$ and $\boldsymbol{E}_{R}=\left[\begin{array}{llll}\boldsymbol{e}_{P} & \boldsymbol{e}_{P+1} & \cdots & \boldsymbol{e}_{M-q}\end{array}\right]$. Then we can easily show that $\left[\begin{array}{ll}\boldsymbol{E}_{I} & \boldsymbol{E}_{R}\end{array}\right]^{H}\left[\begin{array}{ll}\boldsymbol{E}_{I} & \boldsymbol{E}_{R}\end{array}\right]=\boldsymbol{I}$ and

$$
\operatorname{range}\left\{\boldsymbol{E}_{I}\right\}=\operatorname{range}\left\{A_{I}\right\} \perp \operatorname{range}\left\{\boldsymbol{E}_{R}\right\} \text {. }
$$

It follows from (22) and (23) that the optimal weight vector for the EIC based on the criterion shown in (11) can also be written as

$$
\boldsymbol{w}_{\circ}=\left(\boldsymbol{I}-\boldsymbol{E}_{I} \boldsymbol{E}_{I}^{H}\right) \boldsymbol{a}_{q}\left(u_{1}\right)=\boldsymbol{E}_{R} \boldsymbol{E}_{R}^{H} \boldsymbol{a}_{q}\left(u_{1}\right) .
$$

\section{Analysis of the Statistical Performance}

Consider the output SINR of an EIC with optimal weight vector $\boldsymbol{w}_{\circ}$. Following the derivations presented in [7], it is easy to show that the output signal power is given by

$$
p_{s}=\pi_{1}\left|\boldsymbol{w}_{o}^{H} \boldsymbol{a}_{q}\left(u_{1}\right)\right|^{2}
$$

where $\pi_{1}$ denotes the input power of the desired signal, the array output power due to the interference is given by

$$
p_{i}=\boldsymbol{w}_{o}^{H} \boldsymbol{A}_{I} \boldsymbol{\Phi}_{I} A_{I}^{H} \boldsymbol{w}_{o}
$$

and the corresponding output noise power is given by

$$
p_{n}=\pi_{n} \boldsymbol{w}_{o}^{H} \boldsymbol{w}_{o} .
$$

From (25)-(27), the output SINR of the EIC is thus given by

$$
\operatorname{SINR}_{o}=\frac{p_{s}}{p_{i}+p_{n}}=\frac{\pi_{1}\left|\boldsymbol{w}_{o}^{H} \boldsymbol{a}_{q}\left(u_{1}\right)\right|^{2}}{\boldsymbol{w}_{o}^{H} \boldsymbol{A}_{I} \boldsymbol{\Phi}_{I} A_{I}^{H} \boldsymbol{w}_{o}+\pi_{n} \boldsymbol{w}_{o}^{H} \boldsymbol{w}_{o}} .
$$

In practice, the number of signal sources $P$, the background noise power $\pi_{n}$, and the ensemble correlation matrix $R$ required for implementing the EIC are not available and usually estimated from the received data snapshots. Using the first $K$ data snapshots, we obtain the estimate $P$ for the number of signal sources based on the AIC or MDL criterion presented by [11]. Moreover, implementing the AIC or MDL criterion requires performing the EVD of the corresponding data correlation matrix. Therefore, $\pi_{n}$ can be estimated by utilizing the eigenvalue method of [12] during the same estimation process. Let the estimated value be denoted as $\hat{\pi}_{n}$. Then, the next $L$ data snapshots are used to compute the sample correlation matrix $\hat{\boldsymbol{R}}$ as follows:

$$
\hat{\boldsymbol{R}}=\frac{1}{L} \sum_{l=1}^{L} \boldsymbol{x}_{b}\left(t_{l}\right) \boldsymbol{x}_{b}\left(t_{l}\right)^{H}
$$

to replace $\boldsymbol{R}$, where $\boldsymbol{x}_{b}\left(t_{l}\right)$ is the data vector taken at the time instant $t_{l}$. The correlation matrix $\boldsymbol{R}_{w}$ of (21) is then replaced by

$$
\hat{\boldsymbol{R}}_{w}=\hat{\boldsymbol{R}}+\hat{\pi}_{n} \boldsymbol{\Omega} .
$$

It is appropriate to assume that $\hat{\pi}_{n}$ and $\hat{\boldsymbol{R}}$ are independent in this case. Thus, (22) becomes

$$
\hat{\boldsymbol{R}}_{w} \hat{\boldsymbol{e}}_{i}=\hat{\gamma}_{i} \hat{\boldsymbol{e}}_{i}
$$

where $\hat{\gamma}_{1} \geq \hat{\gamma}_{2} \geq \cdots \geq \hat{\gamma}_{M-q}$ are the eigenvalues computed based on $\hat{\boldsymbol{R}}_{w} . \hat{\boldsymbol{e}}_{1}, \hat{\boldsymbol{e}}_{2}, \cdots$, and $\hat{\boldsymbol{e}}_{M-q}$ are the corresponding eigenvectors. Next, let the matrices $\hat{\boldsymbol{E}}_{I}=\left[\hat{\boldsymbol{e}}_{1}, \hat{\boldsymbol{e}}_{2}, \cdots, \hat{\boldsymbol{e}}_{P-1}\right]$ and $\hat{\boldsymbol{E}}_{R}=\left[\hat{\boldsymbol{e}}_{P}, \hat{\boldsymbol{e}}_{P+1}, \cdots, \hat{\boldsymbol{e}}_{M-q}\right]$. Then, the optimal weight vector of the EIC under the $L$ finite samples is given by

$$
\hat{\boldsymbol{w}}_{o}=\left(\boldsymbol{I}-\hat{\boldsymbol{E}}_{I} \hat{\boldsymbol{E}}_{I}^{H}\right) \boldsymbol{a}_{q}\left(u_{1}\right)=\hat{\boldsymbol{E}}_{R} \hat{\boldsymbol{E}}_{R}^{H} \boldsymbol{a}_{q}\left(u_{1}\right) .
$$

Based on the first-order perturbation technique presented in [8] for analysis, it is shown in Appendix that the expectation of the EIC's output SINR using finite data snapshots is approximately given by

$$
E\left\{\widehat{\operatorname{SINR}}_{o}\right\} \approx \operatorname{SINR}_{\circ}\left(1-\frac{1}{L} \operatorname{Tr}\left\{\boldsymbol{\Phi}_{I} \boldsymbol{\Psi}_{I}^{-1}\right\} \frac{\boldsymbol{w}_{o}^{H} \boldsymbol{B}^{H} \boldsymbol{B}_{\boldsymbol{w}_{o}}}{\boldsymbol{w}_{o}^{H} \boldsymbol{w}_{o}}\right)
$$

if the input INR is high enough. Assume that the $P-1$ interferers are uncorrelated. Then $\boldsymbol{\Phi}_{I}=\operatorname{diag}\left\{\pi_{2}, \pi_{3}, \cdots, \pi_{P}\right\}$ and $\boldsymbol{\Psi}_{I}=\operatorname{diag}\left\{\left|\nu_{2}\right|^{2} \pi_{2},\left|\nu_{3}\right|^{2} \pi_{3}, \cdots,\left|\nu_{P}\right|^{2} \pi_{P}\right\}$, where $\pi_{i}=$ 
$E\left\{\left|s_{i}(t)\right|^{2}\right\}$ denotes the input power of the $i$ th signal source. Hence, (33) becomes

$E\left\{\widehat{\operatorname{IINR}}_{o}\right\} \approx \operatorname{SINR}_{\circ}\left(1-\frac{1}{L}\left(\sum_{i=2}^{P}\left|\nu_{i}\right|^{-2}\right) \frac{\boldsymbol{w}_{o}^{H} \boldsymbol{B}^{H} \boldsymbol{B} \boldsymbol{w}_{O}}{\boldsymbol{w}_{O}^{H} \boldsymbol{w}_{o}}\right)$.

Equation (33) reveals that the expectation of the output SINR is bounded in the range of

$$
\begin{aligned}
& {\left[\operatorname{SINR}_{O}\left(1-\frac{1}{L} \operatorname{Tr}\left\{\boldsymbol{\Phi}_{I} \boldsymbol{\Psi}_{I}^{-1}\right\} \lambda_{\max }\left\{\boldsymbol{B}^{H} \boldsymbol{B}\right\}\right)\right.} \\
& \left.\operatorname{SINR}_{\circ}\left(1-\frac{1}{L} \operatorname{Tr}\left\{\boldsymbol{\Phi}_{I} \boldsymbol{\Psi}_{I}^{-1}\right\} \lambda_{\min }\left\{\boldsymbol{B}^{H} \boldsymbol{B}\right\}\right)\right]
\end{aligned}
$$

since $\left(\boldsymbol{w}_{O}^{H} \boldsymbol{B}^{H} \boldsymbol{B} \boldsymbol{w}_{O}\right) /\left(\boldsymbol{w}_{O}^{H} \boldsymbol{w}_{O}\right)$ is bounded by the minimal eigenvalue $\lambda_{\min }\left\{\boldsymbol{B}^{H} \boldsymbol{B}\right\}$ and the maximal eigenvalue $\lambda_{\max }\left\{\boldsymbol{B}^{H} \boldsymbol{B}\right\}$ of $\boldsymbol{B}^{H} \boldsymbol{B}$. It follows from (20) that $\lambda_{\max }\left\{\boldsymbol{B}^{H} \boldsymbol{B}\right\}<\kappa$. Moreover, we note from (35) that the lower bound becomes $\operatorname{SINR}_{o}(1-\kappa(P-1) / L)$ if the $(P-1)$ interferers are uncorrelated and $\left|u_{i}-u_{1}\right|$ are not less than $\pi / 3$ for $i=2,3, \cdots, P$. For example, the value of $\kappa$ is equal to four for $u_{1}=0$ and $q=1$. Thus, the expectation of the output SINR of the EIC converges with a rate at least equal to $1-4(P-1) / L$ when $\left|u_{i}\right|$ are not less than $\pi / 3$ for $i=2,3, \cdots, P$. In the following, we consider two special cases to further simplify the result of (34) under the assumption of $M \geq 2 q$.

\section{A. Single Interferer}

Here, let $u_{1}=0$ and $q=1$. Define the function

$$
g_{M-1}(u)=\frac{\sin ((M-1) u / 2)}{(M-1) \sin (u / 2)} .
$$

After performing some necessary algebraic manipulations, we can obtain

$$
\begin{aligned}
\boldsymbol{w}_{o}^{H} \boldsymbol{w}_{O}= & (M-1)\left(1-g_{M-1}^{2}\left(u_{2}\right)\right) \\
\boldsymbol{w}_{o}^{H} \boldsymbol{B}^{H} \boldsymbol{B w}_{O}= & 2+g_{M-1}^{2}\left(u_{2}\right)\left[2+4(M-2) \sin ^{2}\left(\frac{u_{2}}{2}\right)\right] \\
& -4 g_{M-1}^{2}\left(u_{2}\right) \cos \left(\frac{M-2}{2} u_{2}\right)
\end{aligned}
$$

and

$$
\left|\nu_{2}\right|^{-2}=\left(4 \sin ^{2}\left(\frac{u_{2}}{2}\right)\right)^{-1}
$$

\section{B. Multiple Interferers}

In this case, we assume that the interferers and the desired signal are located so that $\boldsymbol{a}_{q}\left(u_{1}\right)^{H} \boldsymbol{a}_{q}\left(u_{i}\right)$ is approximately equal to zero for $i=2,3, \cdots, P$. Then, the optimal weight vector given by (24) is approximately equal to $\boldsymbol{a}_{q}\left(u_{1}\right)$. Hence, we have from (3) and (4) that

$$
\boldsymbol{w}_{o}^{H} \boldsymbol{w}_{o} \approx \boldsymbol{a}_{q}\left(u_{1}\right)^{H} \boldsymbol{a}_{q}\left(u_{1}\right)=M-q
$$

and

$$
\begin{aligned}
\boldsymbol{w}_{O}^{H} \boldsymbol{B}^{H} \boldsymbol{B} \boldsymbol{w}_{O} & \approx \boldsymbol{a}_{q}\left(u_{1}\right)^{H} \boldsymbol{B}^{H} \boldsymbol{B} \boldsymbol{a}_{q}\left(u_{1}\right) \\
& =\sum_{i=0}^{q-1}\left(\left|\sum_{r=0}^{i} b_{i-r} e^{j r u_{1}}\right|^{2}+\left|\sum_{r=0}^{i} b_{q-r} e^{j r u_{1}}\right|^{2}\right) .
\end{aligned}
$$

Further, substituting the $b_{r}$ of (3) into (41) yields

$$
\begin{aligned}
\boldsymbol{w}_{o}^{H} \boldsymbol{B}^{H} \boldsymbol{B} \boldsymbol{w}_{o} & \approx \boldsymbol{a}_{q}\left(u_{1}\right)^{H} \boldsymbol{B}^{H} \boldsymbol{B a}_{q}\left(u_{1}\right) \\
& =2 \sum_{i=0}^{q-1}\left[\sum_{r=0}^{i}(-1)^{r}\left(\begin{array}{l}
q \\
r
\end{array}\right)\right]^{2} .
\end{aligned}
$$

\section{ANALysis of SENSITIVITy to SteERING ANGLE ERROR}

In practice, the signal blocking matrix is designed according to a steering angle $\theta_{0}$, i.e., $u_{1}$ in (3) and (6) is replaced by $u_{0}=2 \pi d \sin \left(\theta_{0}\right) / \lambda$. For the case of correct steering, we have $\theta_{0}=\theta_{1}$ and $\nu_{1}=0$. Now consider that there is a small mismatch between $\theta_{0}$ and $\theta_{1}$. Using the first-order approximation, the blocking factor $\left|\nu_{1}\right|$ is then approximately given by

$$
\left|\nu_{1}\right|=\left|e^{j u_{1}}-e^{j u_{0}}\right|^{q} \approx\left|u_{1}-u_{0}\right|^{q} .
$$

Equation (43) reveals that $\left|\nu_{1}\right|$ can not be zero since $\left|u_{1}-u_{0}\right|$ is not equal to zero. Hence, there will be a leakage due to the desired signal in the blocked data vector. The correlation matrix of (7) is then given by

$$
\boldsymbol{R}=\boldsymbol{A}_{b} \boldsymbol{\Psi}_{b} \boldsymbol{A}_{b}^{H}+\pi_{n} \boldsymbol{B}^{H} B
$$

where $\boldsymbol{\Psi}_{b}=E\left\{\boldsymbol{s}_{b}(t) \boldsymbol{s}_{b}(t)^{H}\right\}$. Therefore, using the proposed technique to construct $\boldsymbol{R}_{w}=\boldsymbol{R}+\pi_{n} \boldsymbol{\Omega}$ and performing the EVD of this matrix as shown in (22) produces the first $P$ principal eigenvalues which are greater than $\kappa \pi_{n}$. Moreover, the subspace spanned by the eigenvectors corresponding to these $P$ eigenvalues is the same as that spanned by $\boldsymbol{A}_{b}$ if $\boldsymbol{\Psi}_{b}$ has full rank.

When the number of interferers is overestimated, the matrix $\boldsymbol{E}_{I}$ will contain more than $P-1$ principal eigenvectors and, hence, range $\left\{\mathbf{A}_{b}\right\} \subseteq \operatorname{range}\left\{\boldsymbol{E}_{I}\right\}$. From (24), the resulting optimal weight vector $\boldsymbol{w}_{O}$ is given by

$$
\boldsymbol{w}_{o}=\left(\boldsymbol{I}-\boldsymbol{E}_{I} \boldsymbol{E}_{I}^{H}\right) \boldsymbol{a}_{q}\left(u_{0}\right)
$$

which is orthogonal to the response vector $\boldsymbol{a}_{q}\left(u_{1}\right)$, i.e., $\boldsymbol{w}_{o}^{H} \boldsymbol{a}_{q}\left(u_{1}\right)=0$ regardless of the input SNR and the value of $q$. As a result, the desired signal will be completely eliminated and, hence, the EIC will completely fail in this case.

Next, consider the situation where the number of interferers is exactly known and the desired signal is uncorrelated with the $P-1$ interferers. Based on (22) and (44), we have

$$
\boldsymbol{R}_{w}=\pi_{b 1} \boldsymbol{a}_{q}\left(u_{1}\right) \boldsymbol{a}_{q}\left(u_{1}\right)^{H}+\boldsymbol{R}_{I}+\kappa \pi_{n} \boldsymbol{I}
$$

where $\pi_{b 1}=\left|\nu_{1}\right|^{2} \pi_{1}$ denotes the power of the desired signal leakage contained in the blocked data vector and $\boldsymbol{R}_{I}$ the correlation matrix due to the interferers. Let the $P-1$ nonzero eigenvalues and the corresponding eigenvectors of $\boldsymbol{R}_{I}$ are given by $\alpha_{1} \geq \alpha_{2} \geq \cdots \geq \alpha_{P-1}>0$ and $\boldsymbol{v}_{\boldsymbol{i}}$, $i=1,2, \cdots, P-1$, respectively. Consider the situation in which the interferers are located far away from the desired signal so that $\boldsymbol{a}_{q}\left(u_{1}\right)^{H} \boldsymbol{a}_{q}\left(u_{i}\right) \approx 0$. The eigenvalues $\gamma_{i}$, which greater than $\kappa \pi_{n}$ and the corresponding eigenvectors $\boldsymbol{e}_{i}$ of $\boldsymbol{R}_{w}$ in this case can thus be approximately given by $\gamma_{i} \approx \alpha_{i}+\kappa \pi_{n}$ and $\boldsymbol{e}_{i} \approx \boldsymbol{v}_{i}$, for $i=1,2, \cdots, P-1, \gamma_{P} \approx(M-q) \pi_{b 1}+\kappa \pi_{n}$ 
(a)

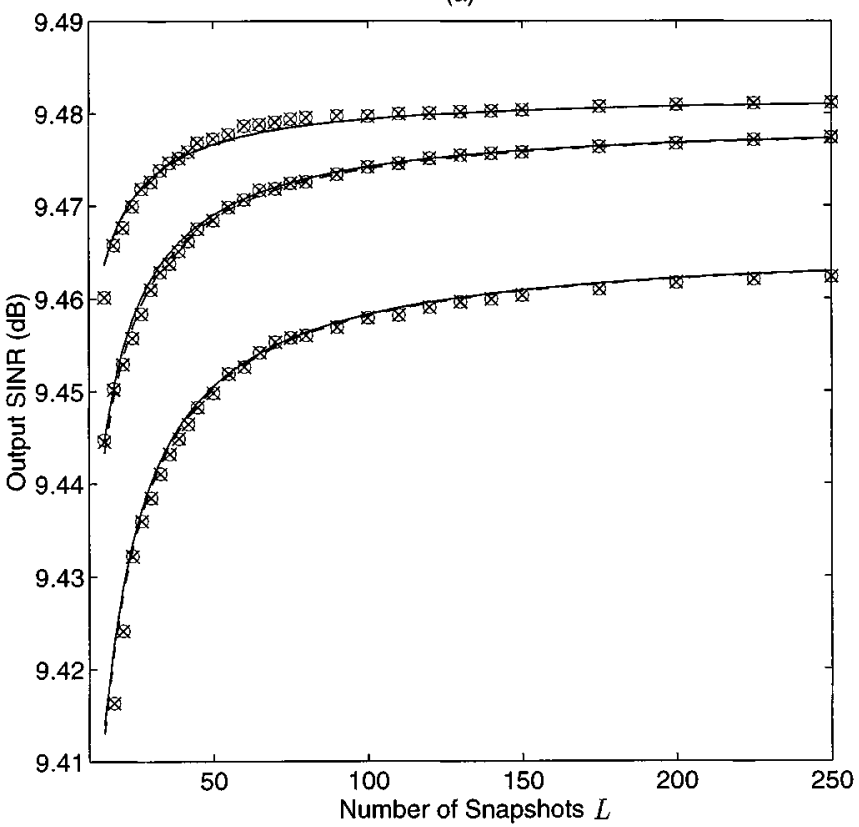

(b)

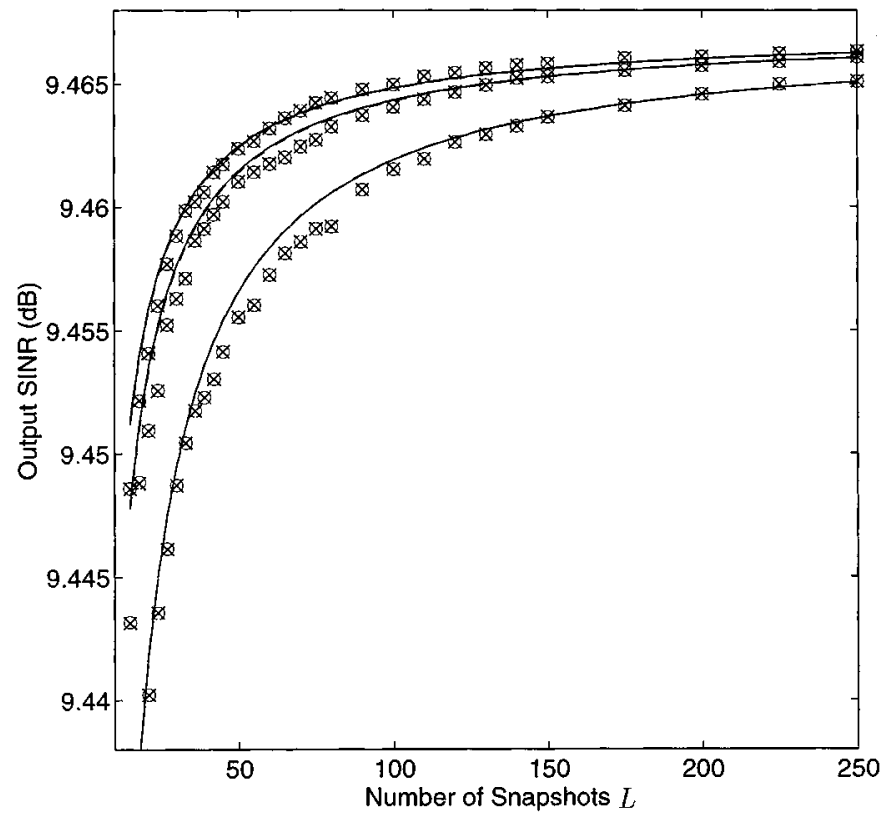

Fig. 1. The results of Example 1. Solid line and dash line: The theoretical results. "x." The proposed technique. "o." The technique of [4]. (a) Output SINR versus the number of snapshots for different number of interferers. (b) Output SINR versus the number of snapshots for blocking matrix with different order.

and $\boldsymbol{e}_{P} \approx \boldsymbol{a}_{q}\left(u_{1}\right) / \sqrt{M-q}$, respectively. Thus, the matrix $\boldsymbol{E}_{I}$ will contain the first $P-1$ principal eigenvectors $\boldsymbol{v}_{i}$, $i=1,2, \cdots, P-1$, when $(M-q) \pi_{b 1}<\alpha_{P-1}$. Consequently, range $\left\{\boldsymbol{E}_{I}\right\} \approx \operatorname{range}\left\{\boldsymbol{A}_{I}\right\}$ and, hence, the EIC will work normally. On the other hand, $\boldsymbol{E}_{I}$ will contain the normalized response vector $e_{P}$ if $(M-q) \pi_{b 1}>\alpha_{P-1}$. From the optimal weight vector given by (45), we can see that the desired signal will be suppressed due to the fact that $\boldsymbol{w}_{o}^{H} \boldsymbol{E}_{I}=0$. As shown by (43) and the fact that $\pi_{b 1}$ is proportional to $\left|\nu_{1}\right|^{2}$, this difficulty could be alleviated by increasing the order $q$ of the signal blocking matrix $\boldsymbol{B}$ if $\left|u_{1}-u_{0}\right|<1$. To look into the effect of $(M-q) \pi_{b 1}>\alpha_{P-1}$, we proceed to investigate the performance of the EIC in the presence of two interferers for the following two cases.

\section{A. Two Highly Correlated and Widely Separated Interferers}

In this case, we assume that two interferers are widely separated so that $\boldsymbol{a}_{q}\left(u_{2}\right)^{H} \boldsymbol{a}_{q}\left(u_{3}\right) \approx 0$ and highly correlated so that the magnitude of their correlation coefficient $\left|\rho_{23}\right| \approx 1$. As shown in [10, pp. 52-55], the eigenvalues of $\boldsymbol{R}_{I}$ are given by

$$
\alpha_{1}=(M-q)\left(\pi_{b 2}+\pi_{b 3}\right)
$$

and

$$
\alpha_{2}=\frac{(M-q) \pi_{b 2} \pi_{b 3}}{\pi_{b 2}+\pi_{b 3}}\left(1-\left|\rho_{2,3}\right|^{2}\right)
$$

where $\pi_{b 2}=\left|\nu_{2}\right|^{2} \pi_{2}$ and $\pi_{b 3}=\left|\nu_{3}\right|^{2} \pi_{3}$ denote the powers of the two interference components contained in the blocked data vector. Hence, $(M-q) \pi_{b 1}>\alpha_{2}$ results in the following performance breakdown threshold for the EIC

$$
\left|\rho_{2,3}\right|^{2}>1-\pi_{b 1}\left(\pi_{b 2}^{-1}+\pi_{b 3}^{-1}\right)
$$

\section{B. Two Weakly Correlated and Closely Separated Interferers}

Here, we assume that two interferers are closely separated so that $\boldsymbol{a}_{q}\left(u_{2}\right)^{H} \boldsymbol{a}_{q}\left(u_{3}\right) \approx M-q$ and weakly correlated so that their correlation coefficient $\rho_{2,3} \approx 0$. Again, following the derivation similar to Section $\mathrm{V}-\mathrm{A}$, we obtain the eigenvalues of $\boldsymbol{R}_{I}$ as follows:

and

$$
\alpha_{1}=(M-q)\left(\pi_{b 2}+\pi_{b 3}\right)
$$

$$
\alpha_{2}=\frac{(M-q) \pi_{b 2} \pi_{b 3}}{\pi_{b 2}+\pi_{b 3}}\left(1-\left|g_{M-q}\left(u_{3}-u_{2}\right)\right|^{2}\right) .
$$

Hence, $(M-q) \pi_{b 1}<\alpha_{2}$ results in the following performance breakdown threshold for the EIC

$$
\left|g_{M-q}\left(u_{3}-u_{2}\right)\right|^{2}>1-\pi_{b 1}\left(\pi_{b 2}^{-1}+\pi_{b 3}^{-1}\right)
$$

Furthermore, if $\left|u_{3}-u_{2}\right|$ is small enough, it is also shown in [10, pp. 52-55] that

$$
\left|g_{M-q}\left(u_{3}-u_{2}\right)\right| \approx 1-\frac{(M-q)^{2}-1}{24}\left(u_{3}-u_{2}\right)^{2} .
$$

From (51), the breakdown threshold of (50) becomes

$$
\left(u_{3}-u_{2}\right)^{2}<\frac{12}{(M-q)^{2}-1} \pi_{b 1}\left(\pi_{b 2}^{-1}+\pi_{b 3}^{-1}\right) .
$$

\section{SIMULATION EXAMPLES AND COMPARISON}

In this section, several simulation examples for confirmation and comparison are presented. The adaptive array considered for all simulations is an $M$-element ULA with interelement spacing equal to half of the signal wavelength.

Example 1: Here, we illustrate the statistical performance of the EIC using an array with $M=10$ sensor elements and a signal blocking matrix $\boldsymbol{B}$ with $q=1$. The desired signal with 
(a)

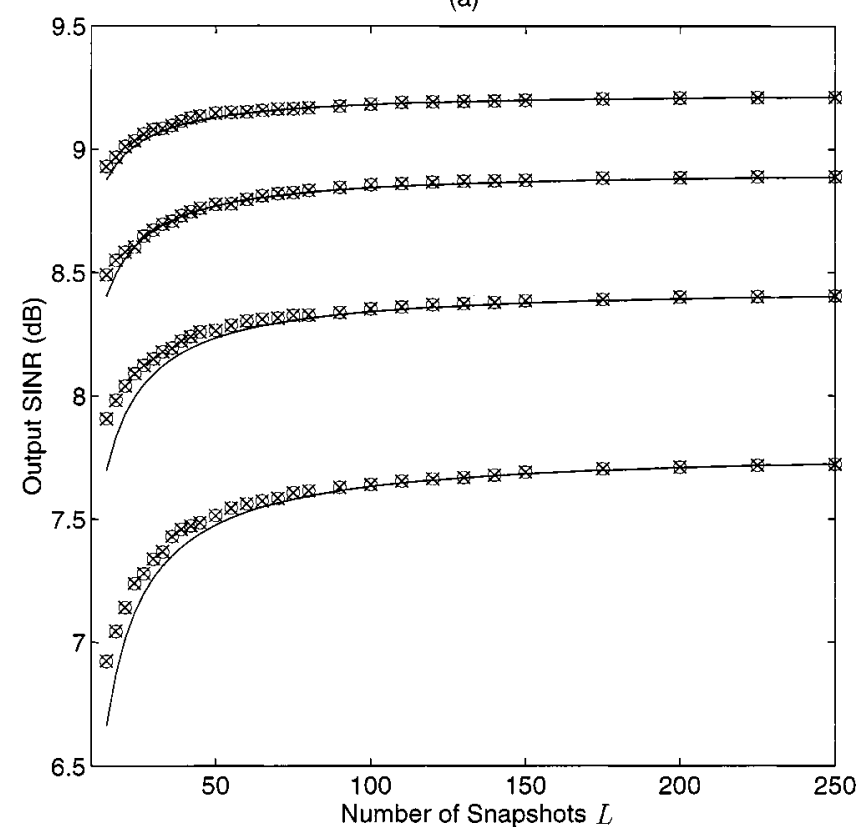

(b)

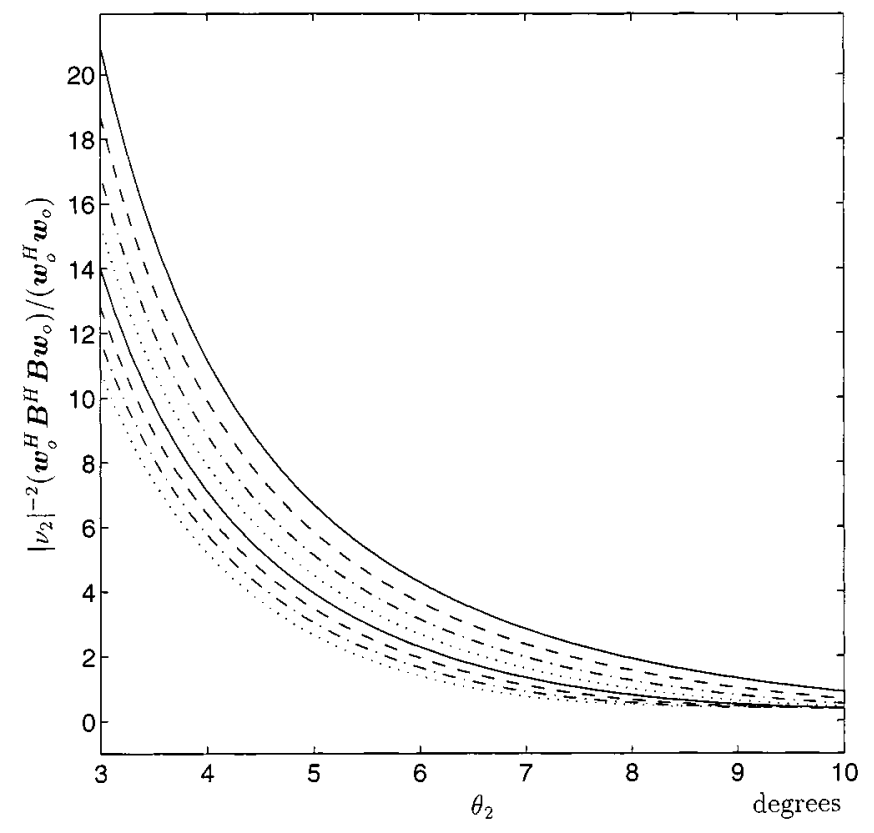

Fig. 2. The results of Example 2. (a) Output SINR versus the number of snapshots. Solid line: The theoretical results. "x." The proposed technique. "o." The technique of [4]. (b) The value of $\left|\nu_{2}\right|^{-2}\left(\omega_{0}^{H} B^{H} \boldsymbol{B} \omega_{0}\right) /\left(\omega_{0}^{H} \omega_{0}\right)$ versus the angle of interference $\theta_{2}$.

input $\mathrm{SNR}=0 \mathrm{~dB}$ is impinging on the array from the broadside, i.e., $\theta_{1}=0$. The first $K=50$ data snapshots are used to estimate the source number $P$ and the noise power $\pi_{n}$ by the procedure described in Section III. Fig. 1(a) shows the expectation of the output SINR versus the number $L$ of snapshots without steering angle error. Three groups of curves from top to the bottom show the simulation results for the three cases, namely one 20-dB interferer with direction angle $\theta_{2}=50^{\circ}$, two uncorrelated 20-dB interferers with direction angles $\theta_{2}=50^{\circ}$ and $\theta_{3}=55^{\circ}$, and three uncorrelated 20-dB interferers with direction angles $\theta_{2}=50^{\circ}, \theta_{3}=55^{\circ}$, and $\theta_{4}=-60^{\circ}$, respectively. For each case, the solid line represents the result com- puted based on (34), while the dash line represents the result computed based on the approximations shown by (40) and (42). In contrast, the curve with " $x$ " represents the result using the proposed EIC, whereas the curve with "o" represents the result using the EIC of [4] based on the average of 100 independent runs. Comparing the results, we observe that the proposed EIC and the EIC of [4] have almost the same performance for this case. Moreover, these simulations confirm the statistical analysis for the proposed EIC presented in Section IV.

Next, we investigate the effect of $q$ on the EIC's performance. Fig. 1(b) shows the expectation of the output SINR versus the number $L$ of snapshots. The desired signal with SNR $=0 \mathrm{~dB}$ is impinging on the array from the broadside, while two uncorrelated interferers with INR $=20 \mathrm{~dB}$ are impinging on the array from $50^{\circ}$ and $-60^{\circ}$, respectively. To make the effective aperture size (which is given by $M-q$ ) and thus the ideal output SINR the same for comparison, we consider three cases with $(M, q)=(12,3),(M, q)=(11,2)$, and $(M, q)=(10,1)$, respectively. Three groups of curves from top to the bottom show the simulation results for the three cases. For each case, the solid line represents the result computed based on (34), while the dash line represents the result computed based on the approximations shown by (40) and (42). In contrast, the curve with " $\mathrm{x}$ " represents the result using the proposed EIC, whereas the curve with "o" represents the result using the EIC of [4] based on the average of 100 independent runs. Comparing the results, we observe that the approximations shown by (40) and (42) are quite appropriate. Again, the proposed EIC and the EIC of [4] have almost the same performance for this case. Moreover, these simulations confirm the statistical analysis for the proposed EIC presented in Section IV.

Example 2: This example illustrates the effect of the angle separation between the desired signal and interference on the EIC's performance. For simplicity, we consider only one interferer with INR $=30 \mathrm{~dB}$ impinging on the array of ten elements from direction angle $\theta_{2}$. The desired signal with input $\mathrm{SNR}=0 \mathrm{~dB}$ is impinging on the array from the broadside. The signal blocking matrix $B$ has order $q=1$. The first $K=50$ data snapshots are used to estimate $P$ and $\pi_{n}$. Fig. 2(a) depicts the expectation of the output SINR versus the number $L$ of snapshots. Four groups of curves from top to the bottom show the simulation results for $\theta_{2}=10^{\circ}, 9^{\circ}, 8^{\circ}$, and $7^{\circ}$, respectively. For each case, the solid line represents the result computed based on the approximations shown by (37)-(39). In contrast, the curve with " $x$ " represents the result using the proposed EIC, whereas the curve with "o" represents the result using the EIC of [4] based on the average of 100 independent runs. We note that the EIC's performance deteriorates as $\theta_{2}$ decreases. Fig. 2(b) plots the value of $\left|\nu_{2}\right|^{-2}\left(\boldsymbol{w}_{o}^{H} \boldsymbol{B}^{H} \boldsymbol{B} \boldsymbol{w}_{o}\right) /\left(\boldsymbol{w}_{o}^{H} \boldsymbol{w}_{o}\right)$ computed from (37)-(39) versus $\theta_{2}$. The curves from top to the bottom show the simulation results for the effective aperture size $M-1$ varying from 10 to 17 . It is clear that the value of $\left|\nu_{2}\right|^{-2}\left(\boldsymbol{w}_{o}^{H} \boldsymbol{B}^{H} \boldsymbol{B} \boldsymbol{w}_{o}\right) /\left(\boldsymbol{w}_{o}^{H} \boldsymbol{w}_{o}\right)$ is a monotone decreasing function of $\theta_{2}$ and, hence, the EIC's performance degrades as $\theta_{2}$ decreases.

Example 3: The sensitivity to steering angle error is investigated. We use an array with ten sensor elements and a signal blocking matrix $B$ with order $q=1$. To avoid the finite sample 
(a)

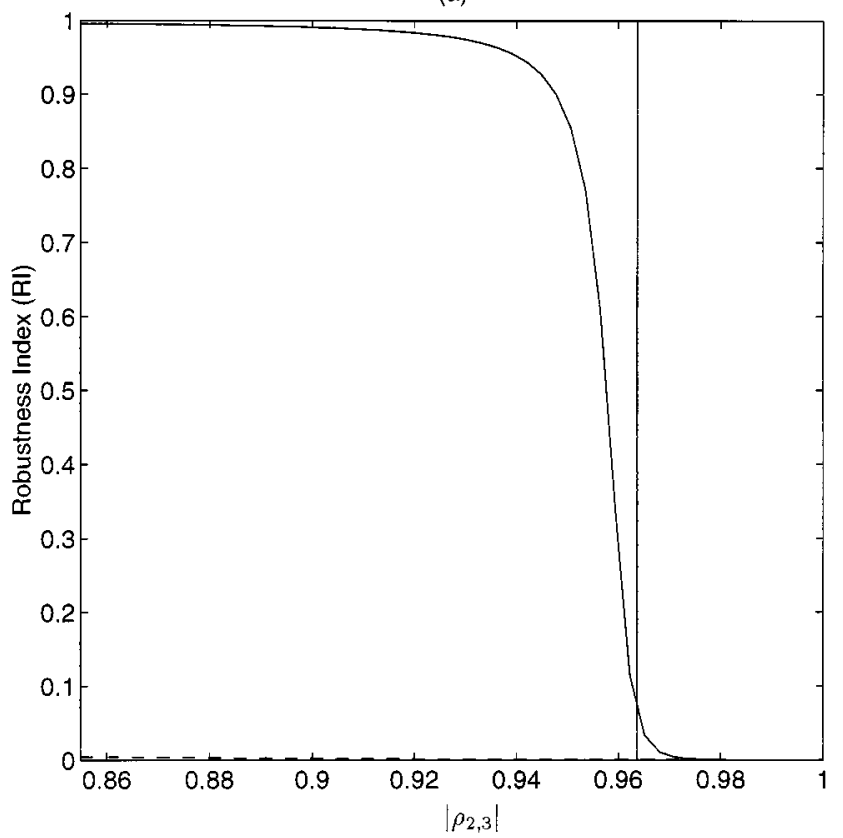

(b)

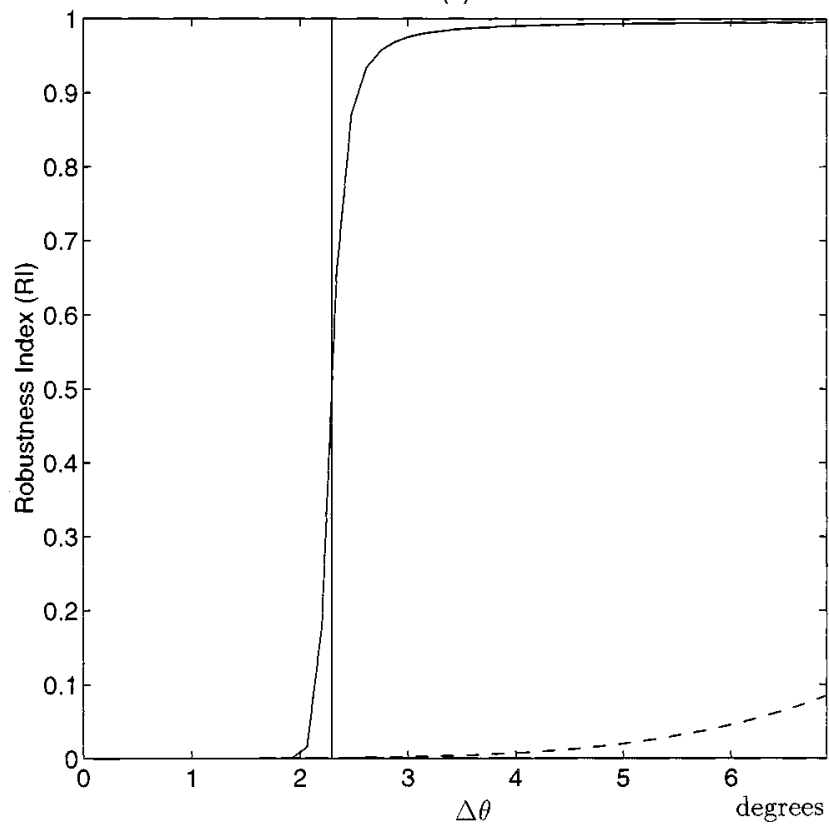

Fig. 3. The results of Example 3. Vertical line: The theoretical breakdown threshold. Solid line and Dash line: RI's of the proposed technique $\left(R I_{1}\right)$ and the technique of [4] $\left(R I_{2}\right)$, respectively. (a) $R I_{1}$ and $R I_{2}$ versus $\left|\rho_{2,3}\right|$. (b) $R I_{1}$ and $R I_{2}$ versus $\Delta \theta$.

effect, the ensemble correlation matrix $\boldsymbol{R}=E\left\{\boldsymbol{x}_{b}(t) \boldsymbol{x}_{b}(t)^{H}\right\}$ is used, where $\boldsymbol{x}_{b}(t)=\boldsymbol{B}^{H} \boldsymbol{x}(t)$ and $\boldsymbol{B}$ is designed based on the steering angle $\theta_{0}$, which is not equal to $\theta_{1}$. To measure the sensitivity of the EIC designed by using the proposed technique to the steering angle error, a robustness index $(R I)$ is introduced as follows:

$R I_{1}=$

Output SINR using (24) with $\boldsymbol{a}_{q}\left(u_{1}\right)$ replaced by $\boldsymbol{a}_{q}\left(u_{0}\right)$ Output SINR using (12) with $\boldsymbol{a}_{q}\left(u_{1}\right)$ replaced by $\boldsymbol{a}_{q}\left(u_{0}\right)$ where $\boldsymbol{E}_{I}$ in (24) is obtained by performing the EVD as shown by (22). In contrast, the $R I$ is defined as

$$
\begin{aligned}
& R I_{2}= \\
& \quad \text { Output SINR using (13) with } \boldsymbol{a}_{q}\left(u_{1}\right) \text { replaced by } \boldsymbol{a}_{q}\left(u_{0}\right) \\
& \text { Output SINR using (12) with } \boldsymbol{a}_{q}\left(u_{1}\right) \text { replaced by } \boldsymbol{a}_{q}\left(u_{0}\right)
\end{aligned}
$$

for measuring the corresponding sensitivity of the EIC designed by using the technique of [4].

First, we consider the case of highly correlated interferers. The desired signal with SNR $=10 \mathrm{~dB}$ is impinging on the array from $\theta_{1}=2^{\circ}$. There are two interferers with INR $=0 \mathrm{~dB}$ and direction angles $\theta_{2}=43^{\circ}$ and $\theta_{3}=-56^{\circ}$, respectively. The steering angle $\theta_{0}=0$. Fig. 3(a) depicts the $R I$ versus the magnitude of the correlation coefficient $\left|\rho_{2,3}\right|$ of these two interferers. The vertical line shows the breakdown threshold computed from (48). For comparison, the results using the EIC of [4] are also plotted. From this figure, we can see that the proposed EIC is very effective for dealing with the situation where steering angle error is encountered.

Next, we consider the case of closely separated interferers. Two uncorrelated interferers with INR $=0 \mathrm{~dB}$ are impinging on the array from $\theta_{2}=55^{\circ}$ and $\theta_{3}=55^{\circ}+\Delta \theta$, respectively. The desired signal with SNR $=7 \mathrm{~dB}$ is impinging on the array from $\theta_{1}=2^{\circ}$. The steering angle is still $\theta_{0}=0^{\circ}$. Fig. 3(b) shows the $R I$ versus $\Delta \theta$. Here, the vertical line shows the breakdown threshold computed from (52). Again, the results using the EIC of [4] are plotted for comparison. We observe that the proposed EIC is more effective than the EIC of [4] against steering angle error. Moreover, it is confirmed by Fig. 3 that the breakdown thresholds shown by (48) and (52) are appropriate theoretical results.

\section{CONCLUSION}

Conventional eigenspace-based interference cancelers (EIC's) like the one of [4] suffer with the lack of performance and sensitivity analyses due to the fact that the noise at the output of its signal blocking matrix is nonwhite. This paper has proposed an EIC and presented the analyses of its performance and sensitivity to steering angle error. We first present a technique to construct a positive definite matrix based on the signal blocking matrix and then use this matrix to compensate the effect of the signal blocking matrix on the sensor noise received by the EIC. Therefore, the interference subspace required for finding the optimal weight vector can be obtained using conventional EVD. This leads to that the performance and sensitivity to steering angle error of the EIC can be analyzed. Computer simulations have confirmed the theoretical results. Moreover, it has been shown by simulations that the performance of the proposed EIC is almost the same as that of [4] in the situations without steering angle error. However, the proposed EIC demonstrates the advantage of possessing robust capabilities against steering angle error over the EIC of [4]. 


\section{APPENDIX}

Here, we show the derivation of the result given by (33). Performing the eigen-decomposition for $\boldsymbol{R}_{w}$, we obtain

$$
\boldsymbol{R}_{w}=\boldsymbol{E}_{I} \boldsymbol{\Lambda}_{I} \boldsymbol{E}_{I}^{H}+\boldsymbol{E}_{R} \boldsymbol{\Lambda}_{R} \boldsymbol{E}_{R}^{H}
$$

where $\boldsymbol{\Lambda}_{I}=\operatorname{diag}\left\{\gamma_{1}, \gamma_{2}, \cdots, \gamma_{P-1}\right\}$ and $\boldsymbol{\Lambda}_{R}=$ $\operatorname{diag}\left\{\gamma_{P}, \gamma_{P+1}, \cdots, \gamma_{M-q}\right\}=\kappa \pi_{n} \boldsymbol{I}$. Similarly, for $\hat{\boldsymbol{R}}_{w}$, we obtain

$$
\hat{\boldsymbol{R}}_{w}=\hat{\boldsymbol{E}}_{I} \hat{\boldsymbol{\Lambda}}_{I} \hat{\boldsymbol{E}}_{I}^{H}+\hat{\boldsymbol{E}}_{R} \hat{\boldsymbol{\Lambda}}_{R} \hat{\boldsymbol{E}}_{R}^{H}
$$

where $\hat{\boldsymbol{\Lambda}}_{I}=\operatorname{diag}\left\{\hat{\gamma}_{1}, \hat{\gamma}_{2}, \cdots, \hat{\gamma}_{P-1}\right\}$ and $\hat{\boldsymbol{\Lambda}}_{R}=$ $\operatorname{diag}\left\{\hat{\gamma}_{P}, \hat{\gamma}_{P+1}, \cdots, \hat{\gamma}_{M-q}\right\}$. The deviation between $\hat{\boldsymbol{R}}_{w}$ and $\boldsymbol{R}_{w}$ due to finite sample effect is given by

$$
\Delta \boldsymbol{R}_{w}=\hat{\boldsymbol{R}}_{w}-\boldsymbol{R}_{w}=\Delta \boldsymbol{R}+\Delta \pi_{n} \boldsymbol{\Omega}
$$

where $\Delta \boldsymbol{R}=\hat{\boldsymbol{R}}-\boldsymbol{R}$ and $\Delta \pi_{n}=\hat{\pi}_{n}-\pi_{n}$. Following the first-order perturbation analysis presented in [8], we can show that

$$
\Delta \boldsymbol{E}_{R}=\hat{\boldsymbol{E}}_{R}-\boldsymbol{E}_{R} \approx-\boldsymbol{R}_{I}^{+} \Delta \boldsymbol{R}_{w} \boldsymbol{E}_{R}
$$

where

$$
\boldsymbol{R}_{I}^{+}=\boldsymbol{E}_{I}\left(\boldsymbol{\Lambda}_{I}-\kappa \pi_{n} \boldsymbol{I}\right)^{-1} \boldsymbol{E}_{I}^{H} .
$$

$\boldsymbol{R}_{I}^{+}$possesses the following properties:

$$
\boldsymbol{R}_{I}^{+}=\boldsymbol{E}_{I}\left(\boldsymbol{A}_{I}^{H} \boldsymbol{E}_{I}\right)^{-1} \boldsymbol{\Psi}_{I}^{-1}\left(\boldsymbol{A}_{I}^{H} \boldsymbol{E}_{I}\right)^{-H} E_{I}^{H}
$$

and

$$
\boldsymbol{R}_{I}^{+} \boldsymbol{R}_{I} \boldsymbol{R}_{I}^{+}=\boldsymbol{R}_{I}^{+}
$$

By substituting (A.4) into (32), the optimal weight vector under finite samples can be approximated by

$$
\hat{\boldsymbol{w}}_{o} \approx \boldsymbol{w}_{o}+\left(\boldsymbol{E}_{R} \Delta \boldsymbol{E}_{R}^{H}+\Delta \boldsymbol{E}_{R} \boldsymbol{E}_{R}^{H}\right) a_{q}\left(u_{1}\right) .
$$

It follows from (A.7) that the output power of the desired signal, the background noise, and the interference are given by

$$
\left\{\begin{array}{l}
\hat{p}_{s}=\pi_{1}\left|\hat{\boldsymbol{w}}_{o}^{H} \boldsymbol{a}_{q}\left(\boldsymbol{u}_{1}\right)\right|^{2} \approx p_{s}+\sum_{k=1}^{3} \Delta p_{s, k}+\text { the first } \\
\hat{p}_{n}=\pi_{n} \hat{\boldsymbol{w}}_{O}^{H} \hat{\boldsymbol{w}}_{O} \approx p_{n}+\sum_{k=1}^{2} \Delta p_{n, k}+\text { the first order term } \\
\hat{p}_{\boldsymbol{i}}=\hat{\boldsymbol{w}}_{O}^{H} \boldsymbol{A}_{I} \boldsymbol{\Phi}_{I} \boldsymbol{A}_{I} \hat{\boldsymbol{w}}_{O} \approx p_{\boldsymbol{i}}+\Delta p_{\boldsymbol{i}}+\text { the first order term }
\end{array}\right.
$$

respectively, where $p_{s}, p_{n}$, and $p_{i}$ denote the corresponding output powers without finite sample effect. Moreover

$$
\begin{aligned}
& \left\{\begin{aligned}
\Delta p_{s, 1}= & \Delta p_{s, 2}^{*}=\pi_{1}\left(\boldsymbol{a}_{q}\left(u_{1}\right)^{H} \Delta \boldsymbol{E}_{R} \boldsymbol{E}_{R}^{H} \boldsymbol{a}_{q}\left(u_{1}\right)\right)^{2} \\
\Delta p_{s, 3}= & 2 \pi_{1}\left(\boldsymbol{a}_{q}\left(u_{1}\right)^{H} \boldsymbol{E}_{R} \Delta \boldsymbol{E}_{R}^{H} \boldsymbol{a}_{q}\left(u_{1}\right)\right) \\
& \cdot\left(\boldsymbol{a}_{q}\left(u_{1}\right)^{H} \Delta \boldsymbol{E}_{R} \boldsymbol{E}_{R}^{H} \boldsymbol{a}_{q}\left(u_{1}\right)\right)
\end{aligned}\right. \\
& \left\{\begin{array}{l}
\Delta p_{n, 1}=\pi_{n} \boldsymbol{a}_{q}\left(u_{1}\right)^{H} \Delta \boldsymbol{E}_{R} \Delta \boldsymbol{E}_{R}^{H} \boldsymbol{a}_{q}\left(u_{1}\right) \\
\Delta p_{n, 2}=\pi_{n} \boldsymbol{a}_{q}\left(u_{1}\right)^{H} \boldsymbol{E}_{R} \Delta \boldsymbol{E}_{R}^{H} \Delta \boldsymbol{E}_{R} \boldsymbol{E}_{R}^{H} \boldsymbol{a}_{q}\left(u_{1}\right)
\end{array}\right.
\end{aligned}
$$

and

$$
\Delta p_{i}=\boldsymbol{a}_{q}\left(u_{1}\right)^{H} \boldsymbol{E}_{R} \Delta \boldsymbol{E}_{R}^{H} \boldsymbol{A}_{I} \boldsymbol{\Phi}_{I} A_{I}^{H} \Delta \boldsymbol{E}_{R} \boldsymbol{E}_{R}^{H} \boldsymbol{a}_{q}\left(u_{1}\right)
$$

denote the second-order perturbation terms, respectively. It is appropriate to neglect the output power due to the interference when the EIC works normally. Accordingly, the output SINR of the EIC can be written as

$$
\widehat{\operatorname{SINR}}_{o}=\frac{p_{s}\left(1+\left(\hat{p}_{s}-p_{s}\right) / p_{s}\right)}{p_{n}\left(1+\left(\hat{p}_{n}-p_{n}\right) / p_{n}+\hat{p}_{i} / p_{n}\right)} .
$$

It is expected that all of the deviation terms $\hat{p}_{s}-p_{s}, \hat{p}_{n}-p_{n}$, and $\hat{p}_{i}-p_{i}$ approach zero as the number of data snapshots increases. Using the first-order approximation when the number of data snapshots is large enough, (A.12) can be approximated by

$\widehat{\operatorname{SINR}_{o}} \approx \operatorname{SINR}_{o}\left(1+\left(\hat{p}_{s}-p_{s}\right) / p_{s}-\left(\hat{p}_{n}-p_{n}\right) / p_{n}-\hat{p}_{i} / p_{n}\right)$.

Since the expectation for each of the first-order terms in (A.8) is zero, the expectation of the output SINR can be approximately given by

$$
\begin{aligned}
& E\left\{\widehat{\operatorname{SINR}}_{o}\right\} \approx \operatorname{SINR}_{o}\left(1+E\left\{\Delta p_{s} / p_{s}\right\}-E\left\{\Delta p_{n} / p_{n}\right\}\right. \\
&\left.-E\left\{\Delta p_{i} / p_{n}\right\}\right)
\end{aligned}
$$

where $\Delta p_{s}=\sum_{k=1}^{3} \Delta p_{s, k}$ and $\Delta p_{n}=\sum_{k=1}^{2} \Delta p_{n, k}$.

In the following, we compute each of the expectation terms $E\left\{\Delta p_{s} / p_{s}\right\}, E\left\{\Delta p_{n} / p_{n}\right\}$, and $E\left\{\Delta p_{i} / p_{n}\right\}$ in (A.14). As shown in (A.3), the deviation $\Delta \boldsymbol{R}_{w}$ is composed of two independent terms, i.e., $\Delta \pi_{n}$ and $\Delta \boldsymbol{R}$. Based on the eigenvalue method of [12] to estimate the noise power, it has been shown that

$$
E\left\{\left|\Delta \pi_{n}\right|^{2}\right\}=\frac{\pi_{n}^{2}}{K(M-P)}
$$

if $K$ data snapshots are used. Next, let $\boldsymbol{R}_{x}=E\left\{\boldsymbol{x}(t) \boldsymbol{x}(t)^{H}\right\}$ and $\hat{\boldsymbol{R}}_{x}=L^{-1} \sum_{l=1}^{L} \boldsymbol{x}\left(t_{l}\right) \boldsymbol{x}\left(t_{l}\right)^{H}$. Then we have

$$
\hat{\boldsymbol{R}}=\boldsymbol{B}^{H} \hat{\boldsymbol{R}}_{x} \boldsymbol{B} \text { and } \Delta \boldsymbol{R}=\boldsymbol{B}^{H} \Delta \boldsymbol{R}_{x} \boldsymbol{B}
$$

where $\Delta \boldsymbol{R}_{x}=\hat{\boldsymbol{R}}_{x}-\boldsymbol{R}_{x}$. Using the result presented in [9], it can be shown that $\Delta \boldsymbol{R}_{x}$ has the following statistical properties

$$
E\left\{\Delta \boldsymbol{R}_{x}\right\}=0
$$

and

$$
\begin{aligned}
& E\left\{\boldsymbol{Q}_{1}^{H} \Delta \boldsymbol{R}_{x} \boldsymbol{Q}_{2} \boldsymbol{Q}_{3}^{H} \Delta \boldsymbol{R}_{x} \boldsymbol{Q}_{4}\right\} \\
& \quad=L^{-1} \operatorname{Tr}\left\{\boldsymbol{Q}_{3}^{H} \boldsymbol{R}_{x} \boldsymbol{Q}_{2}\right\}\left(\boldsymbol{Q}_{1}^{H} \boldsymbol{R}_{x} \boldsymbol{Q}_{4}\right)
\end{aligned}
$$

where $\boldsymbol{Q}_{i}, i=1,2,3,4$ are matrices with appropriate sizes. Substituting (A.4) and (A.3) into (A.9)-(A.11), and using the properties of (A.15) and (A.17), we can obtain the following expectations:

$$
\left\{\begin{aligned}
E\left\{\Delta p_{i}\right\} / p_{n} \approx & E\left\{\Delta p_{i, a}\right\} / p_{n}+E\left\{\Delta p_{i, b}\right\} / p_{n} \\
+ & E\left\{\Delta p_{i, c}\right\} / p_{n} \\
E\left\{\Delta p_{i, a}\right\} / p_{n}= & \left(L \boldsymbol{w}_{o}^{H} \boldsymbol{w}_{o}\right)^{-1} \operatorname{Tr}\left\{\boldsymbol{\Psi}_{I}^{-1} \boldsymbol{\Phi}_{I}\right\} \boldsymbol{w}_{o}^{H} \boldsymbol{B}^{H} \boldsymbol{B} \boldsymbol{w}_{o} \\
E\left\{\Delta p_{i, b}\right\} / p_{n}= & \left(L \boldsymbol{w}_{o}^{H} \boldsymbol{w}_{o}\right)^{-1}\left(\operatorname { T r } \left\{\pi_{n} \boldsymbol{R}_{I}^{+} \boldsymbol{A}_{I} \boldsymbol{\Phi}_{I} \boldsymbol{A}_{I}^{H} \boldsymbol{R}_{I}^{+}\right.\right. \\
& \left.\left.\cdot \boldsymbol{B}^{H} \boldsymbol{B}\right\}\right) \boldsymbol{w}_{o}^{H} \boldsymbol{B}^{H} \boldsymbol{B}_{o} \\
E\left\{\Delta p_{i, c}\right\} / p_{n}= & \left(K(M-P) \boldsymbol{w}_{o}^{H} \boldsymbol{w}_{o}\right)^{-1}\left(\pi_{n} \boldsymbol{w}_{o}^{H} \boldsymbol{\Omega} \boldsymbol{R}_{I}^{+} \boldsymbol{A}_{I} \boldsymbol{\Phi}_{I}\right. \\
& \left.\cdot \boldsymbol{A}_{I}^{H} \boldsymbol{R}_{I}^{+} \boldsymbol{\Omega} w_{o}\right)
\end{aligned}\right.
$$




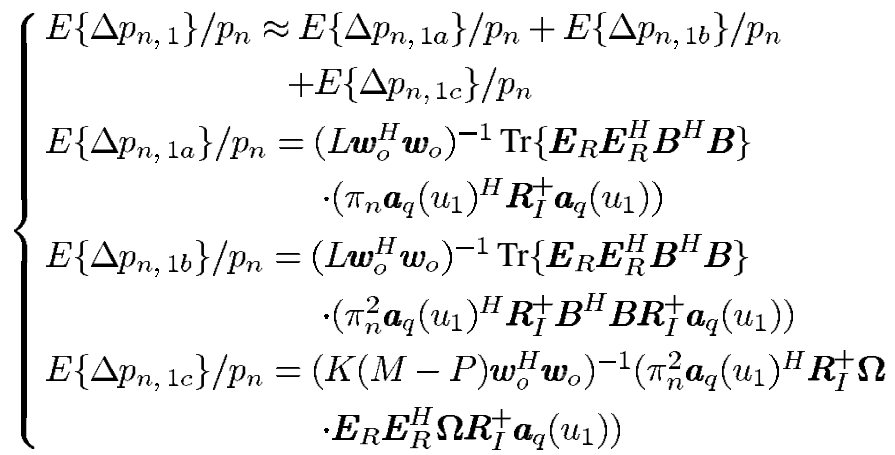

$$
\left\{\begin{aligned}
E\left\{\Delta p_{n, 2}\right\} / p_{n} \approx & E\left\{\Delta p_{n, 2 a}\right\} / p_{n}+E\left\{\Delta p_{n, 2 b}\right\} / p_{n} \\
+ & E\left\{\Delta p_{n, 2 c}\right\} / p_{n} \\
E\left\{\Delta p_{n, 2 a}\right\} / p_{n}= & \left(L \boldsymbol{w}_{o}^{H} \boldsymbol{w}_{o}\right)^{-1} \operatorname{Tr}\left\{\pi_{n} \boldsymbol{R}_{I}^{+}\right\} \boldsymbol{w}_{o}^{H} \\
& \cdot \boldsymbol{B}^{H} \boldsymbol{B} \boldsymbol{w}_{o} \\
E\left\{\Delta p_{n, 2 b}\right\} / p_{n}= & \left(L \boldsymbol{w}_{o}^{H} \boldsymbol{w}_{o}\right)^{-1} \operatorname{Tr}\left\{\pi_{n}^{2} \boldsymbol{R}_{I}^{+} \boldsymbol{B}^{H} \boldsymbol{B} \boldsymbol{R}_{I}^{+}\right\} \boldsymbol{w}_{o}^{H} \\
& \cdot \boldsymbol{B}^{H} \boldsymbol{B} \boldsymbol{w}_{o} \\
E\left\{\Delta p_{n, 2 c}\right\} / p_{n}= & \left(K(M-P) \boldsymbol{w}_{o}^{H} \boldsymbol{w}_{o}\right)^{-1}\left(\pi_{n}^{2} \boldsymbol{w}_{o}^{H} \boldsymbol{\Omega} \boldsymbol{R}_{I}^{+}\right. \\
& \left.\cdot \boldsymbol{R}_{I}^{+} \boldsymbol{\Omega} \boldsymbol{w}_{o}\right)
\end{aligned}\right.
$$

$\left\{\begin{aligned} E\left\{\Delta p_{s, 1}\right\} / p_{s} \approx & E\left\{\Delta p_{s, 1 a}\right\} / p_{s}+E\left\{\Delta p_{s, 1 b}\right\} / p_{s} \\ E\left\{\Delta p_{s, 1 a}\right\} / p_{s}= & \left(L\left(\boldsymbol{w}_{o}^{H} \boldsymbol{w}_{o}\right)^{2}\right)^{-1}\left(\pi_{n} \boldsymbol{w}_{o}^{H} \boldsymbol{B}^{H} \boldsymbol{B} \boldsymbol{R}_{I}^{+} \boldsymbol{a}_{q}\left(u_{1}\right)\right)^{2} \\ E\left\{\Delta p_{s, 1 b}\right\} / p_{s}= & \left(K(M-P)\left(\boldsymbol{w}_{o}^{H} \boldsymbol{w}_{o}\right)^{2}\right)^{-1} \\ & \cdot\left(\pi_{n} \boldsymbol{w}_{o}^{H} \boldsymbol{\Omega} \boldsymbol{R}_{I}^{+} \boldsymbol{a}_{q}\left(u_{1}\right)\right)^{2}\end{aligned}\right.$

and

$$
\left\{\begin{aligned}
E\left\{\Delta p_{s, 3}\right\} / p_{s} \approx & E\left\{\Delta p_{s, 3 a}\right\} / p_{s}+E\left\{\Delta p_{s, 3 b}\right\} / p_{s} \\
+ & E\left\{\Delta p_{s, 3 c}\right\} / p_{s} \\
E\left\{\Delta p_{s, 3 a}\right\} / p_{s}= & 2\left(L\left(\boldsymbol{w}_{o}^{H} \boldsymbol{w}_{o}\right)^{2}\right)^{-1}\left(\pi_{n} \boldsymbol{a}_{q}\left(u_{1}\right)^{H} \boldsymbol{R}_{I}^{+} \boldsymbol{a}_{q}\left(u_{1}\right)\right) \\
& \cdot \boldsymbol{w}_{o}^{H} \boldsymbol{B}^{H} \boldsymbol{B} \boldsymbol{w}_{o} \\
E\left\{\Delta p_{s, 3 b}\right\} / p_{s}=2\left(L\left(\boldsymbol{w}_{o}^{H} \boldsymbol{w}_{o}\right)^{2}\right)^{-1}\left(\pi_{n}^{2} \boldsymbol{a}_{q}\left(u_{1}\right)^{H} \boldsymbol{R}_{I}^{+} \boldsymbol{B}^{H}\right. & \left.\cdot \boldsymbol{B} \boldsymbol{R}_{I}^{+} \boldsymbol{a}_{q}\left(u_{1}\right)\right) \boldsymbol{w}_{o}^{H} \boldsymbol{B}^{H} \boldsymbol{B} \boldsymbol{w}_{o} \\
E\left\{\Delta p_{s, 3 c}\right\} / p_{s}= & 2\left(K(M-P)\left(\boldsymbol{w}_{o}^{H} \boldsymbol{w}_{o}\right)^{2}\right)^{-1} \mid \pi_{n} \boldsymbol{w}_{o}^{H} \boldsymbol{\Omega} \\
& \left.\cdot \boldsymbol{R}_{I}^{+} \boldsymbol{a}_{q}\left(u_{1}\right)\right|^{2} .
\end{aligned}\right.
$$

Without loss of generality, let $\boldsymbol{\Phi}_{I}=c \boldsymbol{\Phi}_{0}$ for some positive number $c$ and positive definite matrix $\boldsymbol{\Phi}_{0}$. According to (A.6), it can be seen that $R_{I}^{+}$is proportional to $c^{-1}$. Moreover, it can be shown from (A.18)-(A.22) that each of the following terms:

$$
\begin{aligned}
& E\left\{\Delta p_{i, b}\right\} / p_{n}, \quad E\left\{\Delta p_{i, c}\right\} / p_{n}, \quad E\left\{\Delta p_{n, 1 a}\right\} / p_{n}, \\
& E\left\{\Delta p_{n, 2 a}\right\} / p_{n}, \quad \text { and } E\left\{\Delta p_{s, 3 a}\right\} / p_{s}
\end{aligned}
$$

is proportional to $c^{-1}$ and each of the following terms:

$$
\begin{array}{ll}
E\left\{\Delta p_{n, 1 b}\right\} / p_{n}, & E\left\{\Delta p_{n, 1 c}\right\} / p_{n}, \quad E\left\{\Delta p_{n, 2 b}\right\} / p_{n}, \\
E\left\{\Delta p_{n, 2 c}\right\} / p_{n}, & E\left\{\Delta p_{s, 1 a}\right\} / p_{s}, \quad E\left\{\Delta p_{s, 1 b}\right\} / p_{s}, \\
E\left\{\Delta p_{s, 3 b}\right\} / p_{s}, & \text { and } E\left\{\Delta p_{s, 3 c}\right\} / p_{s}
\end{array}
$$

is proportional to $c^{-2}$, while only the term $E\left\{\Delta p_{i, a}\right\} / p_{n}$ is fixed and independent of $c$. To get a further simplification, consider the case that $c$ is large enough, i.e., the input INR is high enough such that these terms in (A.23) and (A.24) are negligible as compared to $E\left\{\Delta p_{i, a}\right\} / p_{n}$. Accordingly, the expectation of the output SINR given by (A.14) can be approximated by

$$
E\left\{\widehat{\operatorname{SINR}}_{o}\right\} \approx \operatorname{SINR}_{o}\left(1-\frac{1}{L} \operatorname{Tr}\left\{\boldsymbol{\Psi}_{I} \boldsymbol{\Phi}_{I}^{-1}\right\} \frac{\boldsymbol{w}_{o}^{H} \boldsymbol{B}^{H} \boldsymbol{B} \boldsymbol{w}_{o}}{\boldsymbol{w}_{o}^{H} \boldsymbol{w}_{o}}\right)
$$

\section{REFERENCES}

[1] M. G. Amin, "Concurrent nulling and locations of multiple interference in adaptive antenna arrays," IEEE Trans. Signal Processing, vol. 40, pp. 2658-2663, Nov. 1992.

[2] H. Subbaram and K. Abend, "Interference suppression via orthogonal projections: A performance analysis," IEEE Trans. Antennas Propagat., vol. 41, pp. 1187-1193, Sept. 1993.

[3] B. Friedlander, "A signal subspace method for adaptive interference cancellation," IEEE Trans. Acoust., Speech, Signal Processing, vol. 36, pp. 1835-1845, Dec. 1988

[4] A. M. Haimovich and Y. Bar-Ness, "An eigenanalysis interference canceller," IEEE Trans. Signal Processing, vol. 39, pp. 76-84, Jan. 1991.

[5] Y. Bressler, V. U. Reddy, and T. Kailath, "Optimum beamforming for coherent signals and interference," IEEE Trans. Acoust., Speech, Signal Processing, vol. 36, pp. 833-842, June 1988.

[6] K. Ohnishi and R. T. Milton, "A new optimization technique for adaptive antenna arrays," IEEE Trans. Antennas Propagat., vol. 41, pp. 525-532, May 1993.

[7] T.-J. Shan and T. Kailath, "Adaptive beamforming for coherent signals and interference," IEEE Trans. Acoust., Speech, Signal Processing, vol. ASSP-33, pp. 527-536, June 1985.

[8] F. Li and R. J. Vaccaro, "Unified analysis for DOA estimation algorithms in array signal processing," Signal Processing, vol. 25, pp. 147-169, Nov. 1991.

[9] M. Kaveh and A. J. Barabell, "The statistical performance of the MUSIC and minimum-norm algorithms in resolving plane waves in noise," IEEE Trans. Acoust., Speech, Signal Processing, vol. ASSP-34, pp. 331-341, Apr. 1986.

[10] J. E. Hudson, Adaptive Array Principles. New York: Peter Peregrinus, Ltd., 1981.

[11] M. Wax and T. Kailath, "Detection of signals by information theoretic criteria," IEEE Trans. Acoust., Speech, Signal Processing, vol. ASSP-33, pp. 387-392, Apr. 1985.

[12] P. Stoica, "On estimating the noise power in array processing," Signal Processing, vol. 26, pp. 205-220, Feb. 1992. 


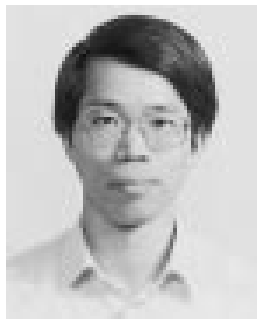

Ju-Hong Lee was born in I-Lan, Taiwan, on December 7, 1952. He received the B.S. degree from the National Cheng-Kung University, Tainan, Taiwan, in 1975, the M.S. degree from the National Taiwan University, Taipei, Taiwan, in 1977, and the $\mathrm{Ph} . \mathrm{D}$. degree from Rensselaer Polytechnic Institute, Troy, NY, in 1984, all in electrical engineering.

From September 1980 to July 1984, he was a Research Assistant and was involved in research on multidimensional recursive digital filtering in the Department of Electrical, Computer, and Systems Engineering at Rensselaer Polytechnic Institute. From August 1984 to July 1986, he was a Visiting Associate Professor and later in August 1986 became an Associate Professor in the Department of Electrical Engineering, National Taiwan University. Since August 1989, he has been a Professor at the same university. He was appointed Visiting Professor in the Department of Computer Science and Electrical Engineering, University of Maryland, Baltimore, during a sabbatical leave in 1996. His current research interests include multidimensional digital signal processing, image processing, detection and estimation theory, analysis and processing of joint vibration signals for the diagnosis of cartilage pathology, and adaptive signal processing and its applications in communications.

Dr. Lee received the Outstanding Research Awards from the National Science Council (NSC) in the academic years of 1988, 1989, and 1991-1994, and the Distinguished Research Awards from the NSC in the academic years of 1998 and 1999.

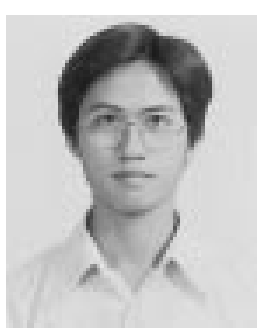

Cheng-Chou Lee was born in Taipei, Taiwan, on July 20, 1969. He received the B.S. and Ph.D. degrees (electrical engineering) from the National Taiwan University, Taipei, Taiwan, in 1991 and 1997, respectively.

His current research interests include the adaptive signal processing, array signal processing, and the signal processing in wireless communication systems. 